\title{
nature/methods
}

\section{Author Correction: Quantitative mapping and minimization of super-resolution optical imaging artifacts}

Siân Culley, David Albrecht, Caron Jacobs, Pedro Matos Pereira (D), Christophe Leterrier (D), Jason Mercer and

Ricardo Henriques (D)

Correction to: Nature Methods https://doi.org/10.1038/nmeth.4605, published online 19 February 2018.

In the Software Availability section, the link to https://bitbucket.org/rhenriqueslab/nanoj-squirrel is obsolete. Code is now available at https://github.com/HenriquesLab/NanoJ-SQUIRREL. The link has not been corrected in the original article.

Published online: 19 October 2020

https://doi.org/10.1038/s41592-020-00983-7

() The Author(s), under exclusive licence to Springer Nature America, Inc. 2020

\section{Author Correction: Genetically encoded tags for direct synthesis of EM-visible gold nanoparticles in cells}

Zhaodi Jiang, Xiumei Jin, Yuhua Li D, Sitong Liu, Xiao-Man Liu D, Ying-Ying Wang, Pei Zhao, Xinbin Cai, Ying Liu, Yaqi Tang, Xiaobin Sun, Yan Liu, Yanyong Hu, Ming Li $\mathbb{D}$, Gaihong Cai, Xiangbing Qi $\mathbb{D}$, She Chen, Li-Lin Du $\mathbb{D}$ and Wanzhong $\mathrm{He}$ D

Correction to: Nature Methods https://doi.org/10.1038/s41592-020-0911-z, published online 10 August 2020.

In the version of this article initially published, an image of HeLa cells expressing Mito-acGFP instead of Mito-acGFP-MTn was used in Fig. 5c. This was due to a mistake during manuscript revision. The panel has now been replaced with the version originally seen by the referees that shows mito-acGFP-MTn labeling. The error has been corrected in the PDF and HTML versions of the article.
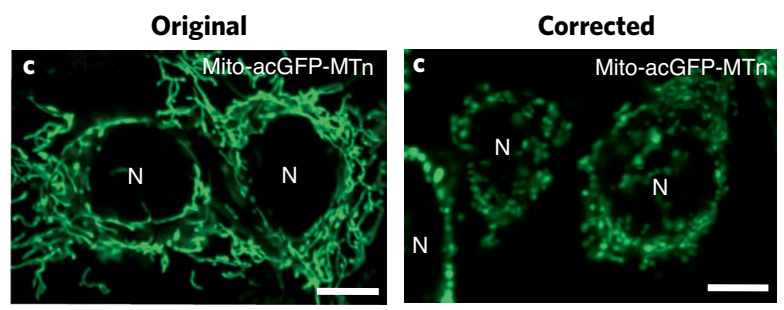

Fig. 5 | Original and corrected.

Published online: 16 October 2020

https://doi.org/10.1038/s41592-020-00996-2

(C) The Author(s), under exclusive licence to Springer Nature America, Inc. 2020 